\title{
ANTECEDENTES DE INOVAÇÕES EM ORGANIZAÇÕES PÚBLICAS DO PODER EXECUTIVO FEDERAL
}

\author{
BACKGROUND OF INNOVATION IN FEDERAL-LEVEL EXECUTIVE BRANCH ENTITIES IN BRAZIL \\ ANTECEDENTES DE INNOVACIONES EN ORGANIZACIONES PÚBLICAS DEL PODER EJECUTIVO FEDERAL
}

\section{Resumo}

A inovação em serviços tem ganhado espaço e é vista por autores como propulsora para o desenvolvimento das organizações, graças ao crescimento da importância do setor para a economia mundial. 0 artigo propõe um construto analítico em que barreiras e facilitadores são antecedentes da inovação, exercendo um papel significativo em sua adoção no setor público, e que existe relação positiva entre antecedentes e tipo de inovação. Para a realização do estudo, foram identificadas variáveis, referenciadas pela literatura, por meio da análise de conteúdo em 286 relatos de experiências de inovação do Concurso de Inovação na Gestão Pública Federal promovido pela Escola Nacional de Administração Pública. Procedeu-se, a posteriori, a análise estatística por meio de regressão logística, identificando a existência de relação significativa entre barreiras e facilitadores da inovação e o tipo de inovação adotado.

Palavras-chave: Inovação em serviços, setor público, antecedentes de inovação, barreiras e facilitadores de inovação, Escola Nacional de Administração Pública.

Cárita Marilhants Silva de Castro - caritamsc@gmail.com

Mestre em Administração pela Universidade de Brasília - Brasília - DF, Brasil

Antônio Isidro-Filho - antonio.isidro.filho@gmail.com

Professor da Universidade de Brasília, Programa de Pós-Graduação em Administração - Brasília - DF, Brasil

Sueli Menelau - suelimenelau@gmail.com

Professora da Universidade Federal de Pernambuco - Caruaru - PE, Brasil

Antonio Sergio Araujo Fernandes - asaferna@outlook.com

Professor da Universidade Federal da Bahia, Escola de Administração, Núcleo de Pós-Graduação em Administração - Salvador - BA, Brasil

Submetido 22.08.2016. Aprovado 13.01.2017

DOI: http://dx.doi.org/10.12660/cgpc.v22n71.63851 


\section{Abstract}

Innovation in services has gained space and several authors view it as a driver of organization development, thanks to the growth of the sector's importance in the world economy. The present article proposes an analytical construct in which barriers and facilitators are antecedents of innovation, playing a significant role in its adoption in the public sector. It also shows a positive relationship between antecedents and type of innovation. For this study, we obtained literature-based variables through content analysis of 286 reports of innovation experiments from the Innovation Competition in Federal Public Management, which was promoted by ENAP. We used logistic regression to conduct a posteriori statistical analysis and found a significant relationship between innovation barriers and facilitators and the type of innovation adopted.

Keywords: Innovation in services, public sector, background of innovation, barriers and facilitators of innovation, ENAP.

\section{Resumen}

La innovación en los servicios ha ganado terreno y es vista por autores como propulsora del desarrollo de las organizaciones, gracias a la creciente importancia del sector para la economía mundial. El artículo propone un constructo analítico en el que las barreras y los facilitadores son antecedentes de la innovación que juegan un papel importante en su adopción en el sector público, y que existe una relación positiva entre los antecedentes y el tipo de innovación. Para la realización del estudio se identificaron variables con referencias en la literatura, mediante el análisis de contenido de 286 informes de experiencias de innovación del Concurso de Innovación en la Gestión Pública Federal, promovido por la ENAP (Escola Nacional de Administracão Pública). A posteriori, se procedió a un análisis estadístico, mediante regresión log ística, que idéentificó la existencia de una relación significativa entre las barreras y los facilitadores de la innovación y el tipo de innovación adoptada.

Palabras clave: Innovación en servicios, sector público, antecedentes de innovación, barreras y facilitadores de la innovación, ENAP.

\section{INTRODUÇÃO}

A relevância dos estudos sobre inovação no setor público é grande ao se considerar a importância de seus serviços para economias e sociedades (Grugulis \& Haynes, 2014; Vargas, Bohrer, Ferreira, \& Moreira, 2013). Inovação no setor público, da mesma forma que nos demais setores, é fruto de um processo entre a capacidade de inovar, a necessidade de inovar e, por que não dizer?, a criatividade. Em estudos realizados na esfera subnacional do Estado brasileiro, como o de Klumb e Hoffmann (2016), sobre o sistema judiciário (em nível estadual), o de Costa e Azeredo (2005), sobre o sistema de educação (em nível municipal), e o de Pinho, Santana e Cerqueira (1997), sobre diversas formas de parceria perpetradas entre o governo local e a sociedade, é possível verificar que o papel da Administração Públi- ca não se limita à mera observação: ela atua em ações e programas radicais, de impacto, que fazem emergir uma cadeia de inovação na qual estão comprometidos seus próprios recursos. Nesse mesmo sentido, Anttiroiko, Bailey e Valkama (2011) apontam que esses desafios domésticos são incentivos para os governos inovarem, o que não justificaria a visão de que o setor público não inova.

Com relação aos trabalhos de inovação em serviços (IS), pode-se afirmar que existem estudos importantes para entender o processo de inovação dentro de organizações públicas, como é o caso dos antecedentes da inovação (Chen, Tsou, \& Huang, 2009; Coutinho \& Bomtempo, 2007; Gebauer, Krempl, \& Fleisch, 2008; Tien \& Berg, 2007). O objetivo principal dos estudos relacionados aos antecedentes da inovação é o de buscar compreender o quanto certo fenômeno influencia, 
como um facilitador, ou inibe, atuando como uma barreira, a capacidade de inovação de uma organização (Panizzon, Milan, \& Toni, 2013).

No Brasil, o interesse pela área de inovação no setor público tem uma literatura vasta, embora nem sempre tenha como foco explícito o tema serviços. Dar conta dessa literatura foge ao escopo deste trabalho, que tem um objetivo mais específico, entretanto destacamos o esforço realizado por pesquisadores e estudiosos em várias subáreas da gestão pública em investigar a inovação no setor público brasileiro. Destaca-se, nessa área, um grupo de autores que trataram de inovação no setor público, tais como Spink (2003), Pinho e Jacobi (2006), Farah (2000), Klering e Andrade (2006), entre outros. Eles investigaram o tema inovação no setor público e coordenaram um grupo de estudos e pesquisa que promoveu a premiação "Experiências de Inovação em Gestão Pública e Cidadania", realizada pela Fundação Getulio Vargas em parceria com a Fundação FORD e o Banco Nacional de Desenvolvimento Econômico e Social (BNDES). Esse programa, que esteve ativo durante 10 anos, entre 1995 e 2005, premiava e difundia experiências inovadoras de governos subnacionais brasileiros que eram capazes de estimular $\mathrm{e}$ promover a cidadania.

Este trabalho tem como objetivo analisar a influência de barreiras e facilitadores em inovações de organizações do Poder Executivo Federal. A unidade de análise da investigação são as experiências de inovação presentes no Concurso de Inovação da Escola Nacional de Administração Pública (ENAP), realizado em parceria com o Ministério do Planejamento, Orçamento e Gestão (MPOG) entre 1996 (ano de início do concurso) e 2014, quando completou 18 edições. Promovido anualmente, o concurso da ENAP tem como objetivo incentivar a implementação e a disseminação de práticas inovadoras na gestão pública, por meio da premiação e divulgação dessas iniciativas (Oliveira, Santana, \& Gomes, 2014).

O concurso da ENAP é importante no que se refere à memória das inovações ocorridas em organizações públicas no Brasil nos últimos 20 anos (Ferrarezi \& Amorim, 2007). O concurso premia os casos de inovação por representarem o compromisso dos servidores envolvidos no processo, que se dedicam a repensar a prestação do serviço público com a adoção de inovações, entendidas como mudanças em práticas anteriores, por meio da incorporação de novos elementos na gestão pública ou de uma nova combinação dos mecanismos de gestão existentes, que produzam resultados positivos para 0 serviço público e para a sociedade (Ferrarezi \& Amorim, 2007; Oliveira et al., 2014).

O artigo é composto de três seções, além desta introdução e de uma seção de conclusão. A seção a seguir trata do referencial teórico-conceitual do trabalho, que são antecedentes de IS no setor público. A seção 3 trata dos procedimentos metodológicos. A seção 4 apresenta os resultados a partir da análise empírica realizada. E a última, a seção 5 , traz algumas considerações à guisa de conclusão.

\section{INOVAÇÃO EM SERVIÇOS E ANTECE- DENTES DE INOVAÇÃO NO SETOR PÚ- BLICO}

Diversos autores trabalham o conceito de 
serviços (Gadrey, 1987; Hill, 1977; Zarifian, 2007), sendo o mais adotado o elaborado por Hill (1977), o qual defende que serviços são mudanças na condição de determinadas realidades, oriundas da ação de um agente econômico a pedido de outro agente que possui interesse e controla essa realidade. Para Gadrey (2000), existem três características na definição de serviço sob uma perspectiva técnica, que o diferenciariam de bens: (i) o serviço é visto como um produto que se encerra no momento em que é produzido, tendo como característica principal sua imaterialidade; (ii) o serviço é concebido como o resultado da cooperação e interação entre produtor e cliente-usuário, a fim de alcançar o resultado desejado; e (iii) entre as principais características do serviço, destacam-se a impossibilidade de transporte e estocagem. Fruto de evolução da abordagem de serviço, a noção do produto final, oriundo de sua prestação, refere-se ao conjunto de características tangíveis e intangíveis que dependem da interação entre provedores de serviços e seus usuários em determinado mercado (Gallouj, 2002; Gallouj \& Weinstein, 1997; Hauknes, 1998; Sundbo \& Gallouj, 1998).

Djellal e Gallouj (2012) propõem que a prestação do serviço inclua a mobilização simultânea de características técnicas (materiais e imateriais) e de competências (internas e externas) para produzir características de serviços. Para Gallouj e Weinstein (1997), "uma vez aceita a representação proposta do produto, a inovação pode ser definida como qualquer mudança que altere um ou mais termos de um ou mais vetores das características (de qualquer tipo - técnica, de serviço ou de competência)" (p. 547). Esses autores propõem tipos de inovação (radical, de melhoria, incremental, recombinativa, de formalização e ad hoc), como resultado da dinâmica das características específicas de serviços e da representação genérica de produto. Pode-se afirmar que a IS constitui a adição de novos elementos ou a combinação de elementos existentes, passíveis de reprodução, que resulta em novas soluções ou em novas formas de prestação de um serviço (Sundbo \& Gallouj, 1998).

Assim, a inovação no setor público pode ser vista tanto como uma modificação substancial, qualitativa ou quantitativa, em técnicas ou práticas anteriores, quanto como uma nova estrutura organizacional ou um novo sistema administrativo, um novo plano ou programa pertencente a membros de uma organização, resultando em um novo produto, serviço ou prática nova para o estado da arte (ou nova, pelo menos, para o contexto organizacional em que se encontra) (Beinare \& McCarthy, 2011; Damanpour \& Wischnevsky, 2006; Røste, 2005). O principal objetivo da inovação no serviço público é otimizar os recursos disponíveis, por meio de formas inovadoras de gestão e organização, gerando maiores benefícios à sociedade, que é a usuária de seus serviços (Soares, 2009).

A inovação é uma ferramenta para melhorar o desempenho organizacional do Estado e garantir sua existência, assegurar que o usuário tenha suas necessidades atendidas ao procurar o órgão público (Costa \& Azeredo, 2005; Klumb \& Hoffmann, 2016; Schlesinger et al., 2008), e, por fim, possibilitar uma participação direta entre prestador (Estado) e usuário (cidadão) em processos de exercício de cidadania, com ganhos significativos para a população como um todo (Mendonça \& Falcão, 2016; Menelau, Vieira, \& Fernandes, 
2016; Pinho et al. 1997).

Lima e Vargas (2012) salientam que, nos estudos de IS, em especial no setor público, um tema ainda polêmico é a tipologia de inovação a ser adotada, pois não há um consenso na literatura. Mas, apesar de críticas e falta de consenso, o modelo de Gallouj e Savona (2009) demonstra ser confiável para a análise do setor público, uma vez que se baseia na abordagem integradora, que visa envolver as situações que permeiam o processo de inovação, seja na produção de bens ou na de serviços (Lima \& Vargas, 2012). Panizzon et al. (2013) estabelecem que variáveis antecedentes podem gerar um efeito positivo ou negativo no nível de inovação, dependendo, inclusive, do contexto analisado (Calantone, Harmancioglu, \& Drodge, 2010). Vigoda-Gadot, Shoam, Schwabsky, e Ruvio (2008) investigaram a inovação no setor público analisando a relação existente entre antecedentes e inovação na perspectiva do cidadão. Seus resultados revelaram que: (i) os antecedentes que exercem maior influência nos resultados da inovação são a capacidade de resposta, liderança e visão; (ii) a inovação impacta positiva ou negativamente a confiança e a satisfação do usuário com a Administração Pública; e (iii) a forma como usuários veem a organização influencia a sua imagem, afetando a confiança e a satisfação deles com a prestação do serviço público. Por sua vez, Menelau et al. (2016) estudaram essa mesma relação sob a perspectiva do núcleo estratégico de gestão de um órgão público e identificaram que o contexto político, o impacto da inovação e a capacidade de (re)combinação do serviço ora atuam como promotores, ora atuam como inibidores da adoção e implementação da inovação.
Para este estudo, os antecedentes organizacionais considerados serão as barreiras e os facilitadores, uma vez que podem influenciar o processo, ao limitar ou propulsar a adoção da inovação (Koch \& Hauknes, 2005; Rego, Pinho, Pedrosa, \& Cunha, 2009). Riege (2005) afirma que, cada vez mais, as organizações vêm buscando manter-se no ambiente por meio da adoção de melhores práticas, tentando superar barreiras e otimizar facilitadores. Por barreiras, devem-se compreender dificuldades que podem gerar conflitos entre diversos enfoques e conduzir o processo à baixa produtividade e qualidade (Segatto-Mendes \& Sbragia, 2002). Facilitadores, por sua vez, são aspectos a melhorar, estimular ou promover o fluxo de conhecimento individual, sócio-organizacional ou tecnológico. Assim como em barreiras, alguns autores utilizam outros termos para se referirem aos facilitadores, tais como ativadores ou alavancas (Martini \& Pellegrini, 2005; Okunoye \& Karsten, 2002; Yeh, Lai, \& Ho, 2006).

\section{PROCEDIMENTOS METODOLÓGICOS}

A pesquisa é definida como um estudo explicativo que visa ilustrar aspectos envolvidos na inovação de serviços no setor público, por meio da criação de uma base de dados e estudo da relação entre as variáveis, fundamentado em pressupostos da literatura. As edições do concurso de inovação promovido pela ENAP escolhidas para coleta dos dados representam um horizonte temporal de 15 anos. A opção por selecionar da quarta até a décima nona edição deu-se pela identificação de que as primeiras edições não se enquadravam em um padrão de descrição, contendo apenas um relato breve das inova- 
ções. Para tanto, apoiou-se em Flick (2009). A avaliação dos resultados é realizada por um comitê julgador composto por especialistas em gestão pública, do Instituto Polis, do Instituto Hélio Beltrão e de órgãos da Administração Pública Federal, como MPOG, Serviço Federal de Processamento de Dados (Serpro) e ENAP (Lemos, 2000).

A população consiste em 432 relatos de experiências. $O$ método de amostragem é não probabilístico (Moore, 2005), perfazendo um total de 197 , tendo sido acrescidas 89 experiências recebidas, que, pelo método de premiação adotado, não puderam ser premiadas, apesar de classificadas. No to- tal, analisaram-se 286 experiências exitosas do concurso, representando $66,2 \%$ da população. $O$ método de coleta de dados foi a pesquisa documental: em setembro de 2015, coletaram-se relatórios anuais das edições selecionadas (no sitio eletrônico www.enap. gov.br, na seção Publicações); em sequência (março de 2016) foram acrescidas 89 experiências inscritas entre 2006 e 2014 e classificadas. Com a junção das etapas, compôs-se um banco de dados, fruto de análise de conteúdo (Bardin, 2011). Na etapa quantitativa, testou-se a relação entre antecedentes de inovação e o tipo de inovação a partir das variáveis "tipos de inovação", "barreiras" e "facilitadores da inovação" (Quadro 1).

Quadro 1. Protocolo das variáveis de análise

\begin{tabular}{|c|c|c|}
\hline \multicolumn{3}{|c|}{ VARIÁVEIS DEPENDENTES } \\
\hline \multicolumn{3}{|c|}{ TIPOS DE INOVAÇÃO } \\
\hline Variável & Definição & Autor \\
\hline Inovação radical & $\begin{array}{l}\text { Criação de um produto totalmente novo; descreve a criação de um } \\
\text { novo conjunto de características } S=\left\{\left[C^{* *}\right],\left[C^{*}\right],\left[T^{*}\right],\left[T^{* *}\right],\left[Y^{*}\right]\right\}\end{array}$ & $\begin{array}{c}\text { Gallouj e Weinstein (1997) } \\
\text { Djellal, Gallouj, e Miles (2013) }\end{array}$ \\
\hline Inovação de melhoria & $\begin{array}{l}\text { Melhoramento de certas características do produto sem alteração na } \\
\text { estrutura do sistema como um todo }\end{array}$ & $\begin{array}{c}\text { Gallouj e Weinstein (1997) } \\
\text { Djellal, Gallouj, e Miles (2013) }\end{array}$ \\
\hline Inovação incremental & $\begin{array}{l}\text { Mudança marginal no sistema por meio de novos elementos adiciona- } \\
\qquad \text { dos para }[\mathrm{X}] \text { e ou }[\mathrm{Y}] \text { ou pela sua substituição }\end{array}$ & $\begin{array}{c}\text { Gallouj e Weinstein (1997) } \\
\text { Djellal, Gallouj, e Miles (2013) }\end{array}$ \\
\hline Inovação recombinativa & $\begin{array}{l}\text { Reutilização sistemática de certos elementos ou componentes do ser- } \\
\text { viço }\end{array}$ & $\begin{array}{c}\text { Gallouj e Weinstein (1997) } \\
\text { Djellal, Gallouj, e Miles (2013) }\end{array}$ \\
\hline Inovação ad hoc & $\begin{array}{l}\text { Construção interativa de uma solução para um problema particular } \\
\text { colocado por um determinado cliente }\end{array}$ & Gallouj e Weinstein (1997) \\
\hline $\begin{array}{l}\text { Inovação de formali- } \\
\text { zação }\end{array}$ & $\begin{array}{c}\text { Implementação que dá visibilidade e otimiza o grau de padronização } \\
\text { das várias características, dando forma e tornando-as mais concretas } \\
\text { e menos confusas }\end{array}$ & $\begin{array}{c}\text { Gallouj e Weinstein (1997) } \\
\text { Djellal, Gallouj, e Miles (2013) }\end{array}$ \\
\hline \multicolumn{3}{|c|}{ VARIÁVEIS INDEPENDENTES } \\
\hline \multicolumn{3}{|c|}{ Barreiras } \\
\hline Variável & Definição & Autor \\
\hline Conflito de interesses & $\begin{array}{c}\text { Tecnologia, cultura organizacional ou expectativas irrealistas que não } \\
\text { fornecem apoio, cooperação e partilha de conhecimentos }\end{array}$ & $\begin{array}{l}\text { Riege (2005), Rego et al. (2009), } \\
\text { Armbrecht et al. (2001), Barnett, } \\
\text { Vasileiou, Djemil, Brooks, e Young } \\
\text { (2011), Rosendaal (2009) }\end{array}$ \\
\hline
\end{tabular}

Continua... 


\section{ANTECEDENTES DE INOVAÇÕES EM ORGANIZAÇÕES PÚBLICAS dO PODER EXECUTIVO FEDERAL}

\begin{tabular}{|c|c|c|}
\hline Dados e sistemas & $\begin{array}{l}\text { Falta de integração, diversidade ou incompatibilidade de sistemas e } \\
\text { processos de TI e/ou de comunicação }\end{array}$ & $\begin{array}{l}\text { Davis, Bagozzi, e Warshaw (1989), } \\
\text { Riege (2005), Parolin, Vasconcellos, } \\
\text { Volpato, e Laurindo (2013), Rego et } \\
\text { al. (2009) }\end{array}$ \\
\hline Rotinas e processos & $\begin{array}{c}\text { Escassez de estrutura organizacional e atividades, formais e informais, } \\
\text { para compartilhar, refletir e gerar (novo) conhecimento. Muitas áreas } \\
\text { de investigação fragmentadas e diversificadas }\end{array}$ & $\begin{array}{l}\text { Riege (2005), Parolin et al. (2013), } \\
\text { Rego et al. (2009), Leonard-Barton } \\
\text { (1998) }\end{array}$ \\
\hline $\begin{array}{l}\text { Limitação de infraestru- } \\
\text { tura, recursos materiais } \\
\quad \text { e tecnológicos }\end{array}$ & $\begin{array}{l}\text { Limitações geográficas, escassez de recursos, falta de redes sociais } \\
\text { para compartilhamento, falta de apoio técnico e de manutenção ime- } \\
\qquad \text { diata aos sistemas integrados de TI }\end{array}$ & $\begin{array}{l}\text { Riege (2005), Parolin et al. (2013), } \\
\text { Rego et al. (2009), Paghaled, } \\
\text { Shafiezadeh, e Mohammadi (2011) }\end{array}$ \\
\hline Limitação de prazo & $\begin{array}{l}\text { Falta ou limitação tempo para interagir e compartilhar conhecimento, } \\
\qquad \text { atender a prazos e realizar tarefas }\end{array}$ & Riege (2005), Rego et al. (2009) \\
\hline $\begin{array}{l}\text { Limitação de recursos } \\
\text { humanos }\end{array}$ & $\begin{array}{l}\text { Má comunicação verbal/escrita e habilidades interpessoais, falta de } \\
\text { motivação, liderança incipiente, falta de treinamento e familiarização do } \\
\text { funcionário com novos processos e sistemas }\end{array}$ & $\begin{array}{l}\text { Riege (2005), Lin e Lee (2006), } \\
\text { Rego et al. (2009) }\end{array}$ \\
\hline $\begin{array}{l}\text { Limitação financeira e } \\
\text { orçamentária }\end{array}$ & $\begin{array}{l}\text { Restrição financeira, falta de remuneração adequada, de incentivos e } \\
\text { reconhecimento }\end{array}$ & $\begin{array}{l}\text { Rego et al. (2009), Wang, Peters, e } \\
\text { Guan (2006), Armbrecht et al. (2001) }\end{array}$ \\
\hline Resistência & $\begin{array}{l}\text { Falta de confiança e de abertura a novas ideias, relutância ao uso de } \\
\text { sistemas, apreensão ou medo de que a partilha de conhecimento ino- } \\
\text { vador seja uma ameaça, causando perda de propriedade e controle do } \\
\text { conhecimento }\end{array}$ & $\begin{array}{l}\text { Barnett et al. (2011), Riege (2005), } \\
\text { Vigoda-Gadot et al. (2008), Rego et } \\
\text { al. (2009) }\end{array}$ \\
\hline \multicolumn{3}{|c|}{ Facilitadores } \\
\hline Variável & Definição & Autor \\
\hline $\begin{array}{l}\text { Comunicação institu- } \\
\text { cional }\end{array}$ & $\begin{array}{l}\text { Estruturas organizacionais abertas fluidas, planas e descentralizadas } \\
\text { que melhoram a comunicação e o fluxo interfuncional, e integram o } \\
\text { conhecimento e a inovação à visão e missão da organização }\end{array}$ & $\begin{array}{l}\text { Barnett et al. (2011), Rego et al. } \\
\text { (2009), Alcará, Chiara, Rodrigues, } \\
\text { Tomáel, e Piedade (2009) }\end{array}$ \\
\hline $\begin{array}{l}\text { Desenvolvimento de } \\
\text { pessoas e competên- } \\
\text { cias }\end{array}$ & $\begin{array}{l}\text { Qualificação de recursos humanos, liberação de acesso e controle da } \\
\text { informação, promoção da cultura de aprendizagem }\end{array}$ & $\begin{array}{l}\text { Parolin et al. (2013), Rego et al. } \\
\text { (2009), Cho, Li e Su (2007) }\end{array}$ \\
\hline $\begin{array}{l}\text { Disponibilidade de } \\
\text { recursos }\end{array}$ & $\begin{array}{l}\text { Incentivos e mecanismos de recompensa, cooperação e partilha, uti- } \\
\text { lização de sistemas de planejamento, apoio à cultura de pesquisa e } \\
\text { tratamento de informações e adaptações tecnológicas que facilitaram a } \\
\text { implementação da inovação }\end{array}$ & $\begin{array}{l}\text { Rego et al. (2009), Armbrecht et al. } \\
\qquad(2001)\end{array}$ \\
\hline $\begin{array}{l}\text { Legitimação e compro- } \\
\text { metimento }\end{array}$ & $\begin{array}{l}\text { Pouca ocorrência de falhas, comportamento proativo, honestidade, } \\
\text { vontade de aprender e compartilhar conhecimentos e ideias que facili- } \\
\text { taram a implementação da inovação }\end{array}$ & $\begin{array}{l}\text { Lin, Wu e Lu (2011), Rego et al. } \\
\text { (2009), Cho et al. (2007) }\end{array}$ \\
\hline
\end{tabular}

Continua... 
Cárita Marilhants Silva de Castro - Antônio Isidro-Filho - Sueli Menelau - Antonio Sergio Araujo Fernandes

\begin{tabular}{|c|c|c|c|}
\hline $\begin{array}{c}\text { Padronização de dados } \\
\text { e processos }\end{array}$ & $\begin{array}{c}\text { Interface entre disciplinas, tecnologias, unidades de negócios, funções } \\
\text { e empresas, centralização do repositório de informações, utilização de } \\
\text { sistemas para tomada de decisão, conexão entre sistemas e informa- }\end{array}$ & $\begin{array}{c}\text { Rego et al. (2009), Armbrecht et al. } \\
\text { (2001), Davis et al. (1989) }\end{array}$ \\
\hline Trabalho em equipe facilitaram a implementação da inovação & $\begin{array}{c}\text { Facilidade de comunicação, incentivo ao trabalho em equipe, clima } \\
\text { de confiança, discussão e apoio das lideranças para disseminação de } \\
\text { novos conhecimentos que facilitaram a implementação da inovação }\end{array}$ & Lin et al. (2011), Rego et al. (2009), \\
& & Cho et al. (2007)
\end{tabular}

Outros dados complementares (utilizados como descritivos) foram coletados: nome da organização; cidade; unidade federativa; e região. As variáveis relevantes para o estudo foram agrupadas e divididas em duas dimensões que contemplaram barreiras e facilitadores (independentes) e tipo de inovação (dependente). A triangulação dos dados coletados deu-se pelo método de triangulação do investigador (Azevedo, Oliveira, Gonzalez, \& Abdalla, 2013). Como ferramenta da análise estatística, foi utilizado um modelo de regressão logística do tipo backward stepwise (Wald), assim como a análise de frequências, por meio do pacote estatístico IBM SPSS Statistics, versão 20, para estabelecer quais das variáveis analisadas seriam possíveis determinantes de que tipo de inovação. $O$ nível de significância estatística foi estabelecido em $p<0,05$ (bi-caudal) para todos os testes.

\section{RESULTADOS, DISCUSSÃO E ANÁLISE DOS DADOS}

A análise de conteúdo identificou os seis tipos de inovação abordados por Gallouj e Weinstein (1997), sendo distribuídos em: 112 casos de inovação incremental, 106 casos de inovação de melhoria, 33 casos de inovação radical, 20 casos de inovação por formalização, oito casos de inovação recombinativa e sete casos de inovação do tipo ad hoc. No que se refere à abordagem de inovação de Gallouj e Weinstein (1997), verificou-se que a tipologia de inovação transcende os limites do setor de serviços privados, podendo ser aplicada em organizações públicas de esfera administrativa federal.

Quanto às barreiras e aos facilitadores à adoção de inovação, esses antecedentes também foram identificados nas organizações públicas do Poder Executivo Federal. Das barreiras que emergiram, perceberam-se variáveis como: resistência, limitação de recursos humanos e conflito de interesses. Quanto aos facilitadores, dos seis propostos no protocolo, destacaram-se: trabalho em equipe, legitimação e comprometimento, e desenvolvimento de pessoas e competências. Os resultados da análise de conteúdo mostram que as principais barreiras e os principais facilitadores identificados nos relatos são estreitamente ligados, uma vez que se trata de variáveis individuais (elementos causais que influenciam a ação do indivíduo).

Nesse sentido, Koch e Hauknes (2005) afirmam que barreiras e facilitadores do processo de inovação em organizações públicas raramente são mutuamente exclusivos, e podem ter relações de causa e efeito entre si, em interações complexas, afetadas e configuradas por variáveis tais como: tamanho 
(do setor e da organização), mentalidade de silo, concordância sobre demandas e políticas, comunicação e escassez/abundância de competências (individuais e organizacionais).

Sobre a região onde se concentram as experiências inovadoras, apresentou-se: $64 \%$ no Distrito Federal (64\%), 17,5\% na região Sudeste, $8,4 \%$ na região Sul, $4,9 \%$ na região Nordeste (4,9\%), 3,1\% na Norte e 2,1\% na Centro-Oeste. Observa-se que esse número é justificável, uma vez que o escopo do trabalho é analisar inovações realizadas em organizações do Poder Executivo Federal, que se encontram, em grande maioria, na capital do País. As análises de frequência permitiram identificar quais variáveis pode- riam ser usadas, visto que a literatura, destinada às aplicações das metodologias quantitativas, muitas vezes, tem apontado que, para uma amostra ser representativa, deve abranger uma porcentagem que represente, pelo menos, de 30 a 40 elementos da população (Oliveira \& Gracio, 2005). Assumindo o pressuposto de Oliveira e Gracio (2005) para realização das análises de influência do tipo de inovação e antecedentes da inovação, e posterior discussão dos resultados, consideraram-se três tipos de inovação: incremental $(n=112)$; de melhoria $(n=106)$; e, radical $(n=33)$ (Djellal et al., 2013; Gallouj \& Weinstein, 1997). Também foram verificadas barreiras e facilitadores, para identificar quais poderiam ser utilizadas na análise de regressão logística (Tabela 1).

Tabela 1. Frequência de barreiras e facilitadores

\begin{tabular}{|l|c|c|}
\hline BARREIRAS E FACILITADORES & $\begin{array}{c}\text { Frequên- } \\
\text { cia }\end{array}$ & $\begin{array}{c}\text { Participação } \\
\text { percentual } \\
\text { (\%) }\end{array}$ \\
\hline Barreira - Conflito de Interesses & 57 & 19,9 \\
Barreira - Dados e sistemas & 51 & 17,8 \\
Barreira - Rotinas e processos & 55 & 19,2 \\
Barreiras de infraestrutura, recursos materiais e tecnológi- & & \\
cos & 68 & 23,8 \\
Barreira - Limitação de prazo & 15 & 5,2 \\
Barreira - Limitação de recursos humanos & 76 & 11,9 \\
Barreira - Limitação financeira e orçamentária & 34 & 41,6 \\
Barreira - Resistência & 119 & 119 \\
Facilitador - Comunicação institucional & 64 & 22,4 \\
Facilitador - Desenvolvimento de pessoas e competências & 92 & 32,2 \\
Facilitador - Disponibilidade de recursos & 150 & 52,4 \\
Facilitador - Legitimação e comprometimento & 125 & 43,7 \\
Facilitador - Padronização de dados e processos & 48 & 16,8 \\
Facilitador - Trabalho em equipe & 149 & 52,1 \\
\hline
\end{tabular}


Da análise de frequências de barreiras e facilitadores, apenas a barreira limitação de prazo não atende ao requisito de, no mínimo, 30 elementos (Oliveira \& Gracio, 2005), portanto foi desconsiderada. O ajuste geral do modelo demonstrou-se adequado, uma vez que apresentou resultado significativo $p$ $=0,000$ (Tabela 2$)$.

Tabela 2. Testes de qui-quadrado e valores de significância

\begin{tabular}{|c|c|c|c|c|c|c|c|}
\hline \multicolumn{2}{|c|}{} & B & S.E & Wald & Df & Sig. & OR $^{*}$ \\
\hline Passo 0 & Constante & $-2,037$ & 0,185 & 12,116 & 1 & 0,000 & 0,130 \\
\hline
\end{tabular}

${ }^{*} \mathrm{OR}=$ odds ratio (razão de probabilidade)

A análise a partir da regressão logística foi conduzida com o objetivo de identificar o grau de influência no tipo de inovação a partir da relação entre as variáveis barreiras e facilitadores, e o tipos de inovação incremental, melhoria e radical. $\mathrm{Na}$ análise multivariada, foram consideradas significativas apenas as variáveis que tiveram $p<0,05$. 0 teste da relação entre as variáveis desenvolvimento de pessoas e competências e inovação incremental apresentou relação positiva $(B=0,596)$ e resultado significativo $(p=0,021$ e $O R=1,815)$, demonstrando que o facilitador desenvolvimento de pessoas e competências pode influenciar a inovação incremental. Segundo Gallouj e Weinstein (1997), é plenamente justificável a relação de influência entre essas variáveis, uma vez que, por definição, esse tipo de inovação se configura pela adição de novas características, ou seja, o desenvolvimento de pessoas e competências, que presume a participação mais efetiva das pessoas, tem como base 0 desenvolvimento de novos conhecimentos, novas habilidades e novas atitudes (Bitencourt, 2002).

Ainda sobre a inovação incremental, identificou-se relação de influência com a barreira dados e sistemas: a probabilidade de ocorrer a inovação incremental aumenta em 1,9 vez ao existir a barreira tecnológica de dados e sistemas na organização $(p=0,686$ e $O R=1,985)$. A relação positiva justifica-se por se tratar de uma barreira tecnológica capaz de influenciar a inovação, na medida em que, para a ocorrência da inovação incremental, existe a possibilidade de remoção de características técnicas para o melhoramento do serviço (Djellal \& Gallouj, 2012; Gallouj, 2002). A relação positiva confirma esse elemento como uma barreira, dada sua definição constitutiva (Quadro 1). A penúltima variável identificada como capaz de influenciar o tipo de inovação foi a barreira de infraestrutura, recursos materiais e tecnológicos nos casos de inovação radical. As inovações foram consideradas radicais (classificadas na análise de conteúdo) porque houve alteração significativa das características do serviço prestado. Identifica-se uma relação positiva entre as variáveis $(B=0,999)$, o que corrobora a ideia de que, havendo uma barreira tão substancial quanto a limitação, escassez ou ausência de infraestrutura ou recursos na organização, aumentam-se as chances de haver uma inovação do tipo radical $(O R=2,715)$, que se caracteriza pela criação de um produto totalmente novo (Gallouj \& Weinstein, 1997). 


\section{ANTECEDENTES dE INOVAÇÕES EM ORGANIZAÇÕES PÚBLICAS dO PODER EXECUTIVO FEDERAL}

A última variável identificada como capaz de influenciar o tipo de inovação foi o facilitador desenvolvimento de pessoas e competências nos casos de inovação de melhoria. Desenvolvimento de pessoas e competências apresenta, na análise, uma relação negativa com a inovação de melhoria $(B=-0,653)$. Pode-se considerar tal relação válida, partindo do pressuposto de que, para a ocorrência desse tipo de inovação, é imperativo haver a necessidade do melhoramento de determinados recursos, como na análise em questão. Portanto, pode-se inferir que a carência de pessoas qualificadas ou da promoção da cultura e aprendizagem organizacional aumenta as chances de ocorrência de uma inovação de melhoria $(\mathrm{OR}=0,521)$.

\section{CONCLUSÕES}

O presente estudo propôs-se a analisar a relação entre tipo de inovação, barreiras e facilitadores, a partir dos relatos de experiências de inovação submetidas, e classificadas ou premiadas, ao Concurso de Inovação na Gestão Pública Federal promovido pela ENAP. A análise de conteúdo realizada nos relatos identificou quais tipos de inovação ocorreram nas organizações públicas pesquisadas, segundo Gallouj e Weinstein (1997), correspondendo a parcela mais representativa aos tipos incremental, de meIhoria e radical. A análise confirma o trabalho de Ferrarezi e Amorim (2007). Os resultados encontrados ainda na etapa qualitativa relativos aos antecedentes de inovação, quanto às barreiras e facilitadores - considerados neste estudo a partir do trabalho desenvolvido por Rego et al. (2009) - foi possível barreiras e facilitadores, uma vez que os relatos se preocupam em apresentar as dificulda- des e os propulsores enfrentados pelas organizações públicas localizadas na esfera federal.

A análise da regressão logística identificou a existência de influência em quatro relações entre barreiras e facilitadores e os três tipos de inovação mais representativos. A insuficiência de significância nas relações das demais variáveis do estudo não implica dizer que não exista contribuição para a inovação, uma vez que as barreiras e facilitadores são fortes obstáculos ou propulsores à implantação de inovação nas organizações (Rego et al., 2009) e devem ser fruto de estudos e análises. $O$ que se percebe é que, em uma análise isolada, essas variáveis, por si mesmas, não são capazes de influenciar o tipo de inovação implementada.

Cabe uma observação no que se refere aos estudos de antecedentes de inovação, uma vez que estes são realizados em organizações do setor privado, o que faz com que boa parte da literatura adotada para a construção do trabalho se refira a esse lócus. Entretanto, como mencionado na introdução deste trabalho, a área de estudo de inovação no setor público brasileiro é vasta, e vem, há pelo menos 20 anos, investigando experiências em governos subnacionais capazes de mostrar que o setor público brasileiro é criativo e dinâmico, e demonstra inovação em várias áreas. Uma possível inferência que fazemos aqui com relação à relevância deste trabalho é que ele pode inserir-se nesse rico e amplo debate já iniciado há algum tempo por importantes autores como Spink (2003), Pinho e Jacobi (2006), Farah (2000), Klering e Andrade (2006), entre outros.

Apesar dos resultados e conclusões obti- 
dos neste trabalho, vale ressaltar que se faz necessária a realização de outros estudos com o intuito de testar novas e diferentes relações entre outras variáveis. O estudo da relação entre as demais variáveis, o tipo de inovação e o desempenho do serviço público pós-inovação permitirá identificar se a inovação realmente incide de maneira positiva não apenas para a solução de problemas, mas também para a melhoria do processo, impactando, assim, em resultados de longo prazo.

\section{REFERÊNCIAS}

Alcará, A. R., Chiara, I. G. D., Rodrigues, J. L., Tomáel, M. I., \& Piedade, V. C. H. (2009). Fatores que influenciam o compartilhamento do conhecimento. Perspectivas em Ciência da Informação, 14(1), 170-191. doi:10.1590/ S1413-99362009000100012

Anttiroiko, A. V., Bailey, S. J., \& Valkama, P. (2011). Innovations in public governance in the Western World. In A. V. Anttiroiko, S. J. Bailey, \& P. Valkama (Eds), Innovations in public governance. Innovation in the public sector, 15, 1-22. Amsterdan: IOS Press. doi: 10.3233/978-1-60750-727-7-1

Armbrecht, F. M. R., Chapas, R. B., Chappelow, C. C., Farris, G. F., Friga, P. N., Hartz, C. A., ... Whitwell, G. E. (2001). Knowledge management in research and development. Research Technology Management, 44(4), 28-48. doi:10.1080/08956308.2001.1167143 8

Azevedo, C. E. F., Oliveira. L. G. L., Gonzalez, R. K., \& Abdalla, M. M. (2013). A estratégia de triangulação: Objetivos, possibilidades, limitações e proximidades com o pragmatis- mo. IV Encontro de Ensino e Pesquisa em Administração e Contabilidade. ENPQ, Brasília, DF.

Bardin, L. (2011). Análise de conteúdo. São Paulo, SP: Edições 70.

Barnett, J., Vasileiou, K., Djemil, F., Brooks L., \& Young T. (2011). Understanding innovators' experiences of barriers and facilitators in implementation and diffusion of healthcare service innovations: A qualitative study. BMC Health Services Research, 11(342), 1-12. doi:10.1186/1472-6963-11-342

Beinare, D., \& McCarthy, M. (2011). Civil society organizations, social innovation and health research in Europe. European Journal of Public Health, 22(6), 889-893. doi:10.1093/ eurpub/ckr152

Bitencourt, C. (2002). A gestão de competências gerenciais e a contribuição da aprendizagem organizacional: A experiência de três empresas australianas. XXVI Encontro Nacional da Associação Nacional dos Programas de Pós Graduação em Administração (ENANPAD). Anpad, Curitiba, PR.

Calantone, R., Harmancioglu, N., \& Drodge, C. (2010). Inconclusive innovation "returns": A meta- analysis of research on innovation in new product development. Journal of Product Innovation Management, 27(7), 1065-1081. doi:10.1111/j.1540-5885.2010.00771.x

Chen, J., Tsou, H. T., \& Huang, A. Y. (2009). Service delivery innovation: Antecedents and impact on firm performance. Journal of Service Research, 12(1), 36-55. doi:10.1177/1094670509338619 


\section{ANTECEDENTES dE INOVAÇÕES EM ORGANIZAÇÕES PÚBLICAS dO PODER EXECUTIVO FEDERAL}

Cho, N., Li, G. Z., \& Su, C. (2007). An empirical study on the effect of individual factors on knowledge sharing by knowledge type. Journal of Global Business and Technology, 3(2), 1-15.

Costa, B. L. D., \& Azeredo, T. M. A. (2005). Inovação, eficiência e eficácia em políticas sociais: Reflexões a partir do Programa Bolsa Escola de Belo Horizonte. Cadernos Gestão Pública e Cidadania, 10(46), 81-107. doi:10.12660/cgpc.v10n46.44040

Coutinho, P. L. A., \& Bomtempo, J. V. (2007). Inovações de produto em uma empresa petroquímica brasileira. Revista Eletrônica de Administração, 13(3), 529-557.

Damanpour, F., \& Wischnevsky, J. D. (2006). Research on innovation in organizations: Distinguishing innovation-generating from innovation-adopting organizations. Journal of Engineering and Technology Management, 23(4), 269-291. doi:10.1016/j.jengtecman.2006.08.002

Davis, F. D., Bagozzi, R. P., \& Warshaw, P. R. (1989). User acceptance of computer technology: A comparison of two theoretical models. Management Science, 35(8), 9821003. doi:10.1287/mnsc. 35.8 .982

Djellal, F., \& Gallouj, F. (2012). L'innovation dans les services publics. Revue Française D'économie, 2(27), 97-142.

Djellal, F., Gallouj, F., \& Miles, I. (2013). Two decades of research on innovation in services: Which place for public services?. Structural Change and Economic Dynamics, 27, 98-117. doi:10.1016/j.strueco.2013.06.005
Farah, M. F. S. (2000). Governo local, políticas públicas e novas formas de gestão pública no Brasil. Organizações e Sociedade, 7(17), 59-86. doi:10.1590/ S1984-92302000000100005

Ferrarezi, E., \& Amorim. S. (2007). Concurso inovação na gestão pública federal no Brasil: Análise de uma trajetória (1996-2006). Cadernos ENAP, 32. Brasília, DF: ENAP.

Flick, U. (2009). Introdução à pesquisa qualitativa. São Paulo, SP: Artmed.

Gadrey, J.(1987). The double dynamics of services. The Services Industries Journal, 7(4), 125-138. doi:10.1080/02642068700000066

Gadrey, J. (2000). The characterization of goods and services: An alternative approach. Review of Income and Wealth, 46(3), 369387. doi:10.1111/j.1475-4991.2000.tb00848.x

Gallouj, F. (2002). Innovation in the service economy: The new wealth of nations. Cheltenham, United Kingdom: Edward Elgar.

Gallouj, F., \& Savona, M. (2009). Innovation in services: A review of the debate and the research agenda. Journal of Evolutionary Economics, 19(2), 149-172. doi:10.1007/ s00191-008-0126-4

Gallouj, F., \& Weinstein, O. (1997). Innovation in services. Research Policy, 26(4-5), 537-556.

Gebauer, H., Krempl, R., \& Fleisch, E. (2008). Exploring the effect of cognitive biases on customer support services. Creativity and Innovation Management, 17(1), 58-70. doi:10.1111/j.1467-8691.2007.00438.x 
Grugulis, I., \& Haynes, K. (2014). Managing services and the service sector: An introduction. In K. Haynes, \& I. Grugulis (Eds.), Managing services: Challenges and innovation (pp. 1-6). New York, EUA: Oxford.

Hauknes, J. (1998). Services in innovation, innovation in services. STEP Report. Oslo, Norway: STEP Group.

Hill, T. P. (1977). On goods and services. Review of Income and Wealth, 23(4), 315-338. doi:10.1111/j.1475-4991.1977.tb00021.x

Klering, L. R., \& Andrade, J. A. (2006). Inovação na gestão pública: Compreensão do conceito a partir da teoria e da prática. In P. Jacobi, \& J. A. Pinho (Orgs.), Inovação no campo da gestão pública local: Novos desafios, novos patamares (Vol. 1, pp. 77-96). São Paulo, SP: FGV.

Klumb, R., \& Hoffmann, M.G. (2016). Inovação no setor público e evolução dos modelos de administração pública: $O$ caso do TRE-SC. Cadernos Gestão Pública e Cidadania, 21(69), 86-102. doi:10.12660/cgpc. v21n69.53902

Koch, P., \& Hauknes, J. (2005). On innovation in the public sector. Oslo, Norway: Publin Report D20: NIFU, STEP.

Lemos, C. (2000). Inovação na era do conhecimento. Revista Parcerias Estratégicas, Centro de Estudos Estratégicos, 8, 157-179.

Leonard-Barton, D. (1998). Nascentes do saber: Criando e sustentando as fontes de inovação. Rio de Janeiro, RJ: FGV.
Lima, D. H., \& Vargas, E. R. (2012). Estudos internacionais sobre inovação no setor público: como a teoria da inovação em serviços pode contribuir? RAP-Revista de Administração Pública, 46(2), 385-401. doi:10.1590/ S0034-76122012000200003

Lin, H. F., \& Lee, G. G. (2006). Effects of socio-technical factors on organizational intention to encourage knowledge sharing. Management Decision, 44(1), 74-88. doi:10.1108/00251740610641472

Lin, T., Wu, S., \& Lu, C. (2011). Exploring the affect factors of knowledge sharing behavior: The relations model theory perspective. Expert Systems with Applications, 39(1), 751764. doi:10.1016/j.eswa.2011.07.068

Martini, A., \& Pellegrini, L. (2005). Barriers and levers towards knowledge management configurations. Journal of Manufacturing Technology Management, 16(6), 670-681. doi:10.1108/17410380510609500

Mendonça, P., \& Falcão, D. S. (2016). Novo marco regulatório para a realização de parcerias entre Estado e Organização da Sociedade Civil (OSC). Inovação ou peso do passado? Cadernos Gestão Pública e Cidadania, 21(68), 44-62. doi:10.12660/cgpc. v21n68.56484

Menelau, S., Vieira, A. F. B. R., \& Fernandes, A. S. A. (2016). Inovação em serviço de segurança pública no Brasil: Facilitadores e barreiras à inovação nos Postos Comunitários de Segurança do Distrito Federal. Revista Brasileira de Políticas Públicas e Internacionais, 1(1), 24-48.

Moore, D. S. (2005). A estatística básica e 
ANTECEDENTES dE INOVAÇÕES EM ORGANIZAÇÕES PÚBLICAS dO PODER EXECUTIVO FEDERAL

sua prática (3a ed.). Rio de Janeiro, RJ: LTC.

Okunoye, A., \& Karsten, H. (2002). ITI as enabler of knowledge management: Empirical perspective from research organisations in subSaharan Africa. IEEE Computer Society.

Oliveira, E. F. T., \& Gracio, M .C. C. (2005). Análise a respeito do tamanho de amostras aleatórias simples: Uma aplicação na área de ciência da informação. Revista de Ciência da Informação, 6(3).

Oliveira, L. G., Santana, R. L. F., \& Gomes, V. C. (2014). Inovação no setor público: Uma reflexão a partir das experiências premiadas no Concurso Inovação na Gestão Pública Federal. Cadernos Enap, 38, 1-59.

Paghaled. M., Shafiezadeh, E., \& Mohammadi, M. (2011). Information technology and its deficiencies in sharing organizational knowledge. International Journal of Business and Social Science, 2(8), 192-198.

Panizzon, M., Milan, G. S., \& Toni, D. De. (2013). Internacionalização, criatividade organizacional e as capacidades dinâmicas baseadas em conhecimento como determinantes da inovação. RAl-Revista de Administração e Inovação, 10(4), 253-282. doi:10.5773/rai.v10i4.1183

Parolin, S. R. H., Vasconcellos, E., Volpato, M., \& Laurindo, A. M. (2013). Barriers and facilitators of collaborative management in technological innovation projects. Journal of Techonology Management \& Innovation, 8, special issue, 151-164.

Pinho, J. A. G., \& Jacobi, P. (Orgs.) (2006).
Inovação no campo da gestão pública local: Novos desafios, novos patamares. Rio de Janeiro, RJ: FGV.

Pinho, J. A. G., Santana, M. W., \& Cerqueira, S. M. B. (1997). Gestão pública em busca de cidadania: Experiências de inovação em Salvador. Organizações e Sociedade, 4(8), 57-90. doi:10.12660/cgpc.v2n5.43831

Rego, A., Pinho, I., Pedrosa, J., \& Cunha, M. P. (2009). Barriers and facilitators to knowledge management in university research centers: An exploratory study. Management Research: Journal of the Iberoamerican Academy of Management, 7(1), 33-47. doi:10.2753/ JMR1536-5433070103

Riege, A. (2005). Three-dozen knowledge-sharing barriers managers must consider. Journal of Knowledge Management, 9(3), 18-35. doi:10.1108/13673270510602746

Rosendaal, B. (2009). Sharing knowledge, being different and working as a team. Knowledge Management Research \& Practice, 7(1), 4-14. doi:10.1057/kmrp.2008.32

Røste, R. (2005). Studies of innovation in the public sector, a theorical framework. Innovation in public sector, 16. Oslo, Norway: NIFU STEP.

Segatto-Mendes, A. P., \& Sbragia, R. (2002). O processo de cooperação universidade-empresa em universidades brasileiras. Revista de Administração da USP, 37(4), 5871.

Schlesinger, C. C. B., Reis, D. R., Silva, H. F. N., Carvalho, H. G., Sus, J. A. L., Ferrari, J. V., ... Xavier, S. A. P. (2008). Gestão do 
conhecimento na administração pública. Curitiba, PR: Instituto Municipal de Administração Pública (IMAP).

Spink, P. (2003, Dezembro). Inovação na perspectiva dos inovadores: A experiência do Programa Gestão Pública e Cidadania. Cadernos EBAPE.BR, 1(2), 1-13. doi:10.1590/S1679-39512003000200002

Soares, A. V. A. (2009). Inovação no setor público: Obstáculos e alternativas. EUPSE.

Sundbo, J., \& Gallouj, F. (1998). Innovation in services. STEP Report. Oslo, Norway: STEP Group.

Tien, J. M., \& Berg, D. (2007). A calculus for services innovation. Journal of System Science and Engieneering, 16(2), 129-165. doi:10.1007/s11518-007-5041-y

Vargas, E. R., Bohrer, C.T., Ferreira, L. B., \& Moreira, M. F. (2013). A pesquisa sobre inovação em serviços no Brasil: Estágio atual, desafios e perspectivas. Revista de Empreendedorismo e Gestão de Pequenas Empresas, 2(1), 3-21.
Vigoda-Gadot, E., Shoam A., Schwabsky N., \& Ruvio, A. (2008). Public sector innovation for Europe: A multinational eigh-country exploration of citizens' perspectives. Public Administration, 86(2), 307-329. doi:10.1111/ j.1467-9299.2008.00731.x

Wang, J., Peters, H. P., \& Guan, J. (2006). Factors influencing knowledge productivity in German research groups: Lessons for developing countries. Journal of Knowledge Management, 10(4), 113-126. doi:10.1108/13673270610679408

Yeh, Y., Lai, S., \& Ho, C. (2006). Knowledge management enablers: A case study. Industrial Management \& Data Systems, 106(6), 793-810. doi:10.1108/02635570610671489

Zarifian, P. (2007). Valor, organização e competência na produção de serviço: Esboço de um modelo de produção de serviço. In: M. S. Salerno (Org),. Relação de serviço: produção e avaliação. (pp. 97-149). São Paulo, SP: Senac. 